\title{
Fatores predisponentes para o desenvolvimento da lesão por pressão em pacientes idosos: uma revisão integrativa
}

\section{Predisposing factors for the development of pressure injury in elderly patients: an integrative review}

\section{Factores predisponentes para el desarrollo de la lesión por presión en pacientes ancianos: una revisión integrada} Nauã Rodrigues de Souza1, Daniela de Aquino Freire', Marcos Antonio de Oliveira Souza',
Jessica Thamires da Silva Melo², Laísa de Veras dos Santos², Magaly Bushatsky²

\begin{abstract}
RESUMO
O estudo teve como objetivo identificar na literatura científica fatores predisponentes para o surgimento das lesões por pressão (LP) em pacientes idosos. Foi realizada uma revisão integrativa nas bases de dados: Literatura Latino-Americana e do Caribe em Ciências da Saúde, Índice Bibliográfico Espanhol de Ciências da Saúde, Base de Dados de Enfermagem, Medical Literature Analysis and Retrieval System Online e na biblioteca Scientific Electronic Library Online, incluindo publicações nacionais e internacionais, no período de 2006 a 2016. Nos 21 artigos analisados houve predominância de risco para desenvolvimento das LP no sexo feminino, e em 9 artigos a variável avanço da idade estava relacionada aos fatores de risco. Os diagnósticos mais observados para o desenvolvimento das LP foram: acidentes vasculares cerebrais, hipertensão arterial sistêmica, diabetes mellitus, cardiopatias, neuropatias e pneumopatias. Sete artigos relacionam a variável alteração da pele e nove associam o uso de medicamentos com a ocorrência de LP nos idosos. Quanto ao aspecto nutricional, 11 artigos avaliaram a relação com a imobilidade do paciente, pois a má nutrição pode influenciar sua capacidade funcional. Ressalta-se, assim, que as lesões por pressão constituem um sério problema comumente identificável em idosos tanto hospitalizados quanto em cuidado domiciliar e em instituições de longa permanência, especialmente quando eles apresentam comprometimento de sua capacidade funcional, evidenciando, a partir das observações, a relevância para a prática clínica e para o cuidado de enfermagem com ênfase na assistência integral e individualizada.
\end{abstract}

DESCRITORES: Lesão por pressão. Fatores predisponentes. Idoso. Estomaterapia.

\begin{abstract}
The objective of this study was to identify, in the scientific literature, predisposing factors for the appearance of pressure injuries (PI) in elderly patients. An integrative review was carried out in the databases: Literatura Latino-Americana e do Caribe em Ciências da Saúde, Índice Bibliográfico Espanhol de Ciências da Saúde, Base de Dados de Enfermagem, Medical Literature Analysis and Retrieval System Online and Scientific Electronic Library Online, including national and international publications, from 2006 to 2016. In the 21 articles analyzed, being a female was a predominant risk factor for the development of Pl; and in 9 articles, advancing age was a risk factor. The most frequent diagnoses for the development of PI were: strokes, systemic arterial hypertension, diabetes mellitus, heart diseases, neuropathies
\end{abstract}

${ }_{1}^{1}$ Programa Associado de Pós-graduação em Enfermagem da Universidade de Pernambuco/Universidade Estadual da Paraíba - Recife (PE), Brasil. Endereço para correspondência: Rua Leão Coroado, 42 - Boa Vista - CEP: 50060-250 - Recife (PE), Brasil - E-mail: nauan_1@hotmail.com

¿Universidade de Pernambuco - Recife (PE), Brasil.

Artigo recebido em: 14/02/2017 - Aceito para publicação em: 05/07/2017 
and pneumopathies. Seven articles related to the variable of skin alteration and nine associated the use of medications with the occurrence of PI in the elderly. Regarding nutritional aspects, 11 articles evaluated the relationship with patient immobility, since malnutrition may influence the patient's functional capacity. It should be emphasized that PI are a serious problem that is commonly identifiable in hospitalized elderly people, as well as in home care and in long-term care facilities, especially when they present impairment of their functional capacity, showing, according to the observations made, the relevance of clinical practice and nursing care with an emphasis on comprehensive and individualized care.

DESCRIPTORS: Pressure ulcer. Predisposing factors. Elderly. Stomatherapy.

\section{RESUMEN}

El estudio tuvo como objetivo identificar en la literatura científica los factores que predisponen para la aparición de lesiones por presión (LP) en pacientes de edad avanzada. Una revisión integradora en las siguientes bases de datos se llevó a cabo: Literatura Latino-Americana e do Caribe em Ciências da Saúde, Indice Bibliográfico Espanhol de Ciências da Saúde, Base de Dados de Enfermagem, Medical Literature Analysis and Retrieval System Online y Scientific Electronic Library Online, incluyendo publicaciones nacionales e internacionales, de 2006 a 2016 . En los 21 artículos analizados, el factor de riesgo predominante para el desarrollo LP ocurrió en el sexo femenino, y en 9 artículos, la variable edad avanzada estaba relacionada con factores de riesgo. Los diagnósticos más frecuentes para el desarrollo de LP eran: accidente cerebrovascular, hipertensión, diabetes mellitus, cardiopatías, neuropatías y neumopatías. Siete artículos relacionan factores de riesgo para LP en los ancianos con la variable alteración de la piel y 9 asocian al uso de drogas. En cuanto al aspecto nutricional, 11 artículos evaluaron la relación con la inmovilidad del paciente, puesto que la mala nutrición puede influir en la capacidad funcional del paciente. Se enfatiza, así, que las LP son un grave problema comúnmente identificable tanto en pacientes de edad avanzada hospitalizados como en el cuidado domiciliar y en instituciones de larga permanencia, especialmente cuando presentan comprometimiento de su capacidad funcional. Se evidencia, a partir de las observaciones, la pertinencia para la práctica clínica y cuidados de enfermería con énfasis en la atención integral e individualizada.

DESCRIPTORES: Úlcera por presión. Factores predisponentes. Ancianos. Estomaterapia.

\section{INTRODUÇÃO}

Existem vários termos de definição para lesão por pressão (LP), como escaras, úlcera de decúbito, úlcera por pressão e ferida de pressão ${ }^{1}$. As LP são consideradas crônicas e sabe-se que ocorrem por meio da compressão entre uma proeminência óssea e uma superfície durante um período de tempo prolongado, levando à morte celular e, consequentemente, ao aparecimento das feridas ${ }^{2}$.

Isso ocorre a partir de dois determinantes etiológicos críticos: a intensidade e a duração da pressão. Existem, ainda, os fatores extrínsecos: fricção, cisalhamento, umidade, e os intrínsecos: redução e/ou perda da sensibilidade, força muscular e imobilidade. É uma questão complexa que, além de gerar dor, deformidades e terapêutica longa, é de difícil resolutividade. No entanto, se for ofertada uma assistência de qualidade e individual, as consequências podem ser minimizadas e o restabelecimento efetivo ${ }^{3}$.

A LP atinge em torno de $9 \%$ de todos os pacientes internados, sendo a maioria idosos, e cerca de 23\% dos acamados que estão em tratamento residencial ${ }^{4}$. Os idosos são as pessoas mais susceptíveis a desenvolver LP, devido às próprias condições causadas pelo envelhecimento do corpo humano, como a diminuição da espessura da pele, das fibras elásticas e rigidez do colágeno, além da redução do tecido adiposo subcutâneo nos membros, diminuição de capilares da derme, que pode ocasionar a redução do suplemento sanguíneo e a desidratação da pele, os quais são fatores que predispõem ao surgimento das lesões ${ }^{5,6}$.

Em idosos, o envelhecimento ocorre de forma progressiva e irreversível e ocasiona diversas modificações em seu sistema fisiológico ${ }^{6}$. Além do aparecimento de doenças crônico-degenerativas, podem ocorrer restrições motoras, visuais, intelectuais e auditivas em pessoas com idades mais avançadas, reduzindo sua condição de saúde. O envelhecimento proporciona fatores predisponentes para o desenvolvimento e agravamento do estágio inicial da LP e, consequentemente, uma maior probabilidade de surgir uma $\mathrm{LP}^{7}$.

O sucesso na prevenção e na diminuição das LP depende do conhecimento dos fatores predisponentes e da habilidade da equipe de saúde, principalmente da equipe de enfermagem que presta assistência direta aos pacientes de maneira 
individualizada ${ }^{8}$. Contudo, torna-se indispensável abranger os fatores predisponentes individuais e institucionais que influenciam o uso das evidências pelos profissionais, de forma que as estratégias possam ser planejadas e utilizadas ${ }^{9}$.

Desse modo, o enfermeiro, além de se tornar o ator fundamental para avaliação habitual do idoso, deve fornecer medidas de prevenção eficazes para esse agravo. É extremamente essencial que este profissional apresente conhecimento sobre as principais causas e complicações ocasionadas pela LP, distinguindo e classificando o grau de dependência e prescrevendo os cuidados de enfermagem essenciais na prevenção do surgimento da $\mathrm{LP}^{10}$.

As lesões na pele ainda são um problema que desafia os profissionais de saúde, pois mesmo sendo evitáveis, existe um grande número de LP na prática clínica, como é o caso dos pacientes com mobilidade física restrita. Entre as principais consequências desse agravo, podemos citar o aumento do tempo e do custo de internamento hospitalar, impacto negativo no serviço prestado e diminuição no bem-estar dos indivíduos ${ }^{1}$.

O surgimento das LP ocorre em geral de forma rápida e progressiva. As complicações ao indivíduo são diversas, entre elas destacamos: tratamento e reabilitação demorada, alteração na qualidade de vida devido ao surgimento de dor e elevação do risco de mortalidade ${ }^{6}$. Diante dessa problemática abrangente, tanto para os pacientes quanto para os familiares, é importante conhecer e identificar os fatores mais prevalentes para a ocorrência das LP. Sendo assim, o presente estudo objetivou identificar na literatura científica os fatores predisponentes para o surgimento das LP em idosos.

\section{METODOLOGIA}

Trata-se de uma revisão integrativa da literatura, método específico que resume obras empíricas ou teóricas para fornecer uma compreensão mais abrangente de um fenômeno particular, com potencial de apresentar o estado da ciência, contribuir para o desenvolvimento da teoria e ter aplicabilidade direta à prática e à política ${ }^{11}$.

Para ser considerada uma pesquisa, a revisão de literatura deve seguir o mesmo rigor da pesquisa primária. Nesse sentido, foram consideradas as fases de pesquisa:

- identificação do problema e objetivo da pesquisa;

- pesquisa da literatura com foco sobre o tema a ser estudado;
- avaliação dos dados aplicando critérios de inclusão e exclusão;

- análise dos dados extraídos das fontes primárias, características da amostra e método, que, no caso, foi qualquer referência ao conceito de abandono/não adesão e fatores relacionados;

- como última fase, temos a apresentação da síntese, que retrata a construção dos conceitos e seus fatores relacionados ${ }^{11}$.

Buscou-se responder ao seguinte questionamento: quais são os fatores predisponentes descritos na literatura para o desenvolvimento de LP em pacientes idosos?

Os critérios de inclusão adotados para a busca e seleção das publicações foram: artigos publicados em periódicos científicos nacionais e internacionais que abordassem a temática: fatores predisponentes para desenvolvimento de LP em pacientes idosos, divulgados em língua portuguesa, inglesa ou espanhola, no período de 2006 a 2016 e indexados nas bases de dados: Literatura Latino-Americana e do Caribe em Ciências da Saúde (LILACS); Índice Bibliográfico Espanhol de Ciências da Saúde (IBECS), Base de Dados de Enfermagem (BDENF), Medical Literature Analysis and Retrieval System Online (MEDLINE) e na biblioteca Scientific Electronic Library Online (SciELO); disponibilizados na íntegra, diretamente pelo site da base ou pelo Portal Capes; localizáveis por intermédio da combinação dos seguintes descritores cadastrados no Portal de Descritores das Ciências da Saúde (DeCS): "úlceras por pressão", "úlceras de pressão", "fatores predisponentes " e "idoso". Esses descritores foram combinados com os operadores booleanos AND e OR a fim de refinar os estudos de acordo com o tema em questão.

A busca foi realizada de forma ordenada, classificando-se na primeira análise os artigos nas seguintes categorias: fora do período considerado, que não disponibilizavam o texto completo, publicados em mais de uma base (duplicatas) e os selecionados para segunda análise. Posteriormente, realizou-se a leitura criteriosa do título e do resumo de cada publicação a fim de verificar a consonância com a pergunta norteadora da investigação. Quando houve dúvida referente à inclusão ou exclusão do estudo, o mesmo foi lido na íntegra para reduzir o risco de perdas de publicações relevantes ao estudo. Desse modo, aqueles que não abordaram o tema "fatores predisponentes para desenvolvimento de LP em pacientes idosos" foram descartados na segunda análise (Figura 1). 
A coleta de dados se deu entre os meses de julho e agosto de 2016 e contou com o apoio de um instrumento de coleta de dados elaborado no software Microsoft Office Excel 2010, com as seguintes variáveis: título do artigo, autores, periódico, ano de publicação, país de origem do estudo, tipo/abordagem do estudo.

\section{RESULTADOS E DISCUSSÃO}

$\mathrm{Na}$ presente revisão, analisaram-se 21 artigos. Estes apresentaram diferentes características no que se refere aos países em que foram realizados, autores, tipo de estudo e periódico publicado. Essas características são evidenciadas no Quadro 1.

Com base no Quadro 1, foi possível observar, quanto à origem das publicações, que nove artigos eram brasileiros, sendo que cinco foram realizados no nordeste e quatro no sudeste; dois dos Estados Unidos da América, dois da Alemanha e outros com somente uma publicação: China, Turquia, Israel, Portugal e Espanha.
Quanto ao tipo de delineamento metodológico, evidenciou-se que a maioria $(n=7)$ são estudos retrospectivos que buscam uma maior compreensão dos fatores predisponentes para desenvolvimento de LP em idosos. Em relação ao quantitativo de publicações por ano, em 2012 houve cinco publicações, e nos anos de 2015 e 2010, três publicações cada. A revista que mais publicou sobre a temática em questão foi a Revista Acta Paulista de Enfermagem, com três publicações, as demais tiveram apenas uma. Observou-se que 15 artigos foram produzidos exclusivamente por enfermeiros ${ }^{2,3,13,17-20,23,24,26,27,29,30}$; cinco por médicos ${ }^{12,14-16,22}$; e dois conjuntamente por médicos e enfermeiros ${ }^{21,15}$.

\section{Variáveis associadas ao desenvolvimento de lesão por pressão}

O Quadro 2 apresenta a síntese dos resultados dos estudos selecionados, abordando as principais variáveis associadas aos fatores predisponentes ao desenvolvimento das LP em idosos. Em relação ao sexo, os estudos apresentam

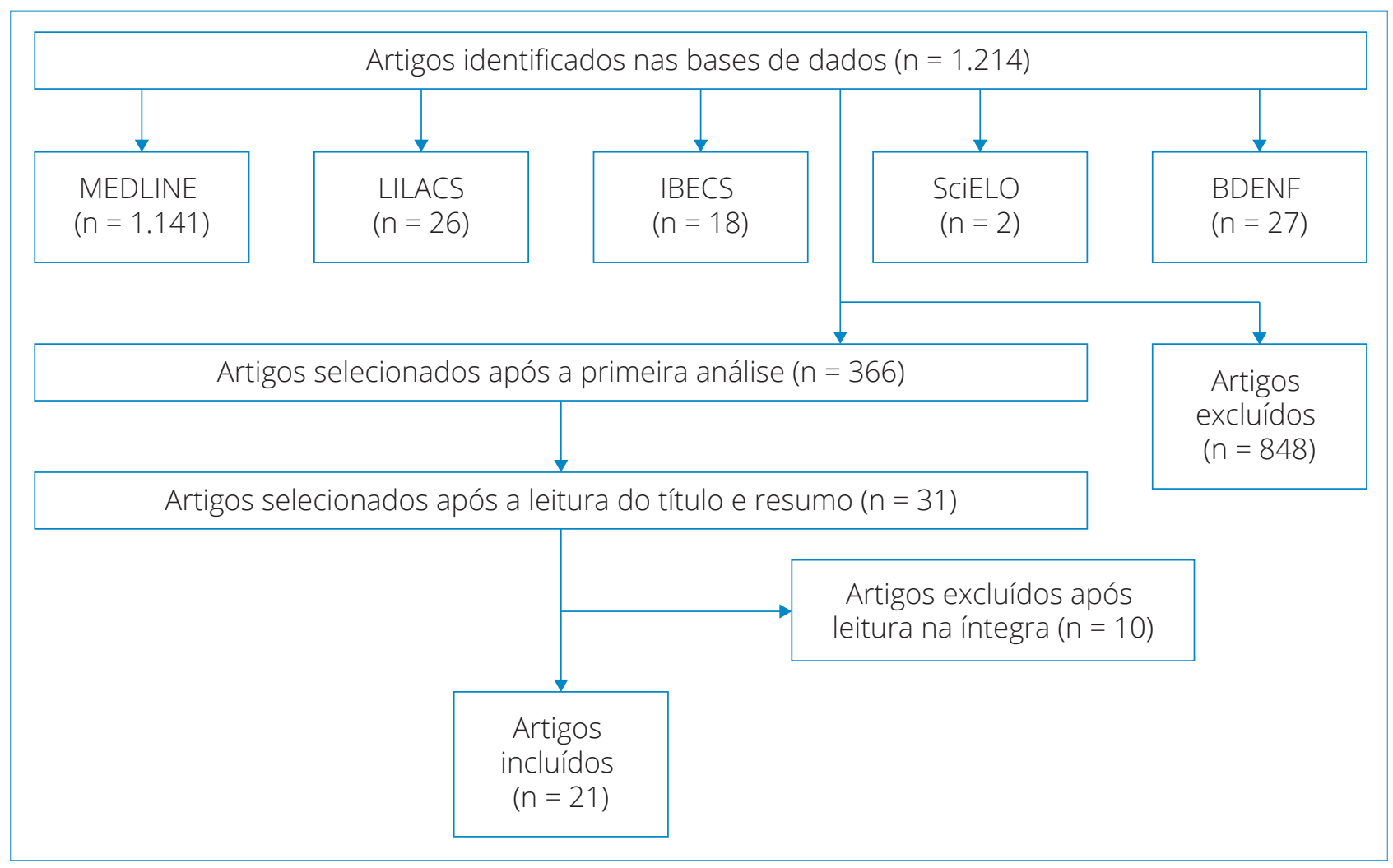

Figura 1. Processo de seleção dos estudos nas bases Literatura Latino-Americana e do Caribe em Ciências da Saúde (LILACS), Medical Literature Analysis and Retrieval System Online (MEDLINE), Scientific Electronic Library Online (SciELO), Índice Bibliográfico Espanhol de Ciências da Saúde (IBECS) e Base de Dados de Enfermagem (BDENF). Recife, Pernambuco, 2017. 
Quadro 1. Caracterização da produção científica sobre os fatores predisponentes para úlcera por pressão em idosos. Recife, Pernambuco, Brasil, 2017.

\begin{tabular}{|c|c|c|c|c|}
\hline Título do artigo & Autores & $\begin{array}{c}\text { Ano de } \\
\text { publicação e } \\
\text { país }\end{array}$ & $\begin{array}{l}\text { Tipo de } \\
\text { estudo }\end{array}$ & Periódico \\
\hline $\begin{array}{l}\text { Systemic factors and mortality in elderly } \\
\text { patients with pressure ulcers }\end{array}$ & $\begin{array}{l}\text { Jaul e } \\
\text { Calderon- } \\
\text { Margalit' }\end{array}$ & $\begin{array}{l}2015 \\
\text { Israel }\end{array}$ & $\begin{array}{l}\text { Estudo } \\
\text { retrospectivo }\end{array}$ & Int Wound J \\
\hline $\begin{array}{l}\text { Prevalência de lesão por fricção e fatores } \\
\text { associados: revisão sistemática }\end{array}$ & $\begin{array}{l}\text { Strazzieri- } \\
\text { Pulido et al. }{ }^{13}\end{array}$ & $\begin{array}{l}2015 \\
\text { Brasil }\end{array}$ & $\begin{array}{l}\text { Revisão } \\
\text { sistemática }\end{array}$ & $\begin{array}{l}\text { Rev Esc } \\
\text { Enferm USP }\end{array}$ \\
\hline $\begin{array}{l}\text { Pressure ulcers and their associated factors } \\
\text { in nursing home inmates }\end{array}$ & Akca et al. ${ }^{14}$ & $\begin{array}{l}2015 \\
\text { Turquia }\end{array}$ & $\begin{array}{l}\text { Revisão } \\
\text { narrativa }\end{array}$ & $\begin{array}{l}\text { J Coll } \\
\text { Physicians } \\
\text { Surg Pak }\end{array}$ \\
\hline $\begin{array}{l}\text { Caracterização e fatores predisponentes } \\
\text { para úlceras por pressão na pessoa idosa } \\
\text { hospitalizada }\end{array}$ & Vieira et al. ${ }^{10}$ & $\begin{array}{l}2014 \\
\text { Brasil }\end{array}$ & $\begin{array}{l}\text { Estudo } \\
\text { de coorte } \\
\text { prospectivo }\end{array}$ & Rev Rene \\
\hline $\begin{array}{l}\text { Obesity and pressure ulcers among nursing } \\
\text { home residents }\end{array}$ & Cai et al. ${ }^{15}$ & $\begin{array}{l}2013 \\
\text { Estados } \\
\text { Unidos da } \\
\text { América }\end{array}$ & $\begin{array}{l}\text { Estudo } \\
\text { retrospectivo }\end{array}$ & Med Care \\
\hline $\begin{array}{l}\text { Hypotension is a risk factor for new pressure } \\
\text { ulcer occurrence in older patients after } \\
\text { admission to an acute hospital }\end{array}$ & $\begin{array}{l}\text { Man e Au- } \\
\text { Yeung }{ }^{16}\end{array}$ & $\begin{array}{l}2013 \\
\text { China }\end{array}$ & $\begin{array}{l}\text { Estudo } \\
\text { de coorte } \\
\text { retrospectivo }\end{array}$ & $\begin{array}{l}\text { J Am Med Dir } \\
\text { Assoc }\end{array}$ \\
\hline $\begin{array}{l}\text { Aplicação da Escala de Braden em } \\
\text { domicílio: incidência e fatores associados } \\
\text { à úlcera por pressão }\end{array}$ & $\begin{array}{l}\text { Freitas e } \\
\text { Alberti }^{17}\end{array}$ & $\begin{array}{l}2013 \\
\text { Brasil }\end{array}$ & $\begin{array}{l}\text { Estudo } \\
\text { de coorte } \\
\text { prospectivo }\end{array}$ & $\begin{array}{l}\text { Acta Paul } \\
\text { Enferm }\end{array}$ \\
\hline $\begin{array}{l}\text { Avaliação da capacidade funcional de } \\
\text { idosos associada ao risco de úlcera } \\
\text { por pressão }\end{array}$ & Aguiar et al. ${ }^{18}$ & $\begin{array}{l}2012 \\
\text { Brasil }\end{array}$ & $\begin{array}{l}\text { Estudo } \\
\text { quantitativo } \\
\text { transversal }\end{array}$ & $\begin{array}{l}\text { Acta Paul } \\
\text { Enferm }\end{array}$ \\
\hline $\begin{array}{l}\text { Avaliação de risco para úlcera } \\
\text { por pressão em idosos acamados } \\
\text { no domicílio }\end{array}$ & $\begin{array}{l}\text { Moraes } \\
\text { et al. }{ }^{19}\end{array}$ & $\begin{array}{l}2012 \\
\text { Brasil }\end{array}$ & $\begin{array}{l}\text { Estudo } \\
\text { longitudinal } \\
\text { prospectivo }\end{array}$ & $\begin{array}{l}\text { Acta Paul } \\
\text { Enferm }\end{array}$ \\
\hline $\begin{array}{l}\text { Risco para úlcera por pressão em } \\
\text { idosos hospitalizados: aplicação da } \\
\text { Escala de Waterlow }\end{array}$ & $\begin{array}{l}\text { Fernandes } \\
\text { et al. }{ }^{20}\end{array}$ & $\begin{array}{l}2012 \\
\text { Brasil }\end{array}$ & $\begin{array}{l}\text { Estudo } \\
\text { exploratório } \\
\text { descritivo }\end{array}$ & $\begin{array}{l}\text { Rev Enferm } \\
\text { UERJ }\end{array}$ \\
\hline $\begin{array}{l}\text { Relación entre el estado nutricional y el } \\
\text { riesgo de presentar úlceras por presión } \\
\text { en pacientes incluidos en el programa de } \\
\text { atención domiciliaria }\end{array}$ & $\begin{array}{l}\text { Sancho } \\
\text { et al. }{ }^{21}\end{array}$ & $\begin{array}{c}2012 \\
\text { Espanha }\end{array}$ & $\begin{array}{l}\text { Estudo } \\
\text { quantitativo } \\
\text { transversal }\end{array}$ & $\begin{array}{l}\text { Atención } \\
\text { Primaria }\end{array}$ \\
\hline $\begin{array}{l}\text { Care-related risk factors for hospital- } \\
\text { acquired pressure ulcers in elderly adults } \\
\text { with hip fracture }\end{array}$ & $\begin{array}{l}\text { Baumgarten } \\
\text { et al. }{ }^{22}\end{array}$ & $\begin{array}{l}2012 \\
\text { EUA }\end{array}$ & $\begin{array}{l}\text { Estudo } \\
\text { de coorte } \\
\text { retrospectivo }\end{array}$ & $\begin{array}{l}\text { J Am Geriatr } \\
\text { Soc }\end{array}$ \\
\hline $\begin{array}{l}\text { Fatores de risco para úlceras de pressão } \\
\text { em idosos de Unidade de Terapia Intensiva }\end{array}$ & $\begin{array}{l}\text { Creutzberg } \\
\text { et al. }{ }^{23}\end{array}$ & $\begin{array}{l}2011 \\
\text { Brasil }\end{array}$ & $\begin{array}{l}\text { Estudo } \\
\text { retrospectivo }\end{array}$ & Enferm Foco \\
\hline $\begin{array}{l}\text { Úlcera por pressão em idosos } \\
\text { institucionalizados: análise da prevalência } \\
\text { e fatores predisponentes }\end{array}$ & Freitas et al. ${ }^{3}$ & $\begin{array}{l}2011 \\
\text { Brasil }\end{array}$ & $\begin{array}{l}\text { Estudo } \\
\text { de coorte } \\
\text { retrospectivo }\end{array}$ & $\begin{array}{l}\text { Rev Gaúcha } \\
\text { Enferm }\end{array}$ \\
\hline
\end{tabular}


Quadro 1. Continuação.

\begin{tabular}{|c|c|c|c|c|}
\hline Título do artigo & Autores & $\begin{array}{l}\text { Ano de } \\
\text { publicação e } \\
\text { país }\end{array}$ & $\begin{array}{l}\text { Tipo de } \\
\text { estudo }\end{array}$ & Periódico \\
\hline $\begin{array}{l}\text { The relationship between malnutrition } \\
\text { parameters and pressure ulcers in hospitals } \\
\text { and nursing homes }\end{array}$ & $\begin{array}{l}\text { Shahin } \\
\text { et al. }{ }^{24}\end{array}$ & $\begin{array}{c}2010 \\
\text { Alemanha }\end{array}$ & $\begin{array}{l}\text { Estudo } \\
\text { quantitativo } \\
\text { transversal }\end{array}$ & Nutrition \\
\hline $\begin{array}{l}\text { Extrinsic risk factors for pressure ulcers } \\
\text { early in the hospital stay: a nested } \\
\text { case-control study }\end{array}$ & $\begin{array}{c}\text { Baumgarten } \\
\text { et al. }{ }^{25}\end{array}$ & $\begin{array}{l}2008 \\
\text { EUA }\end{array}$ & $\begin{array}{l}\text { Estudo de } \\
\text { coorte/caso- } \\
\text { controle }\end{array}$ & $\begin{array}{c}\text { J Gerontol A } \\
\text { Biol Sci Med } \\
\text { Sci }\end{array}$ \\
\hline $\begin{array}{l}\text { Friction and shear highly associated with } \\
\text { pressure ulcers of residents in long-term } \\
\text { care - Classification Tree Analysis (CHAID) of } \\
\text { Braden items }\end{array}$ & $\begin{array}{l}\text { Lahmann } \\
\text { et al. }{ }^{26}\end{array}$ & $\begin{array}{c}2011 \\
\text { Alemanha }\end{array}$ & $\begin{array}{l}\text { Estudo } \\
\text { retrospectivo }\end{array}$ & $\begin{array}{c}\text { J Eval Clin } \\
\text { Pract }\end{array}$ \\
\hline $\begin{array}{l}\text { Pressure ulcer development in } \\
\text { older residents in nursing homes: } \\
\text { influencing factors }\end{array}$ & Kwong et al. ${ }^{27}$ & $\begin{array}{l}2009 \\
\text { China }\end{array}$ & $\begin{array}{l}\text { Estudo } \\
\text { de coorte } \\
\text { prospectivo }\end{array}$ & J Adv Nurs \\
\hline $\begin{array}{l}\text { Riesgos a úlceras por presión según Escala } \\
\text { Norton en una población de ancianos } \\
\text { de un servicio domiciliario de Lima }\end{array}$ & $\begin{array}{c}\text { Eleonora e } \\
\text { Gissela28 }\end{array}$ & $\begin{array}{c}2008 \\
\text { Espanha }\end{array}$ & $\begin{array}{l}\text { Estudo } \\
\text { descritivo } \\
\text { transversal }\end{array}$ & $\begin{array}{c}\text { Rev Enferm } \\
\text { Herediana }\end{array}$ \\
\hline $\begin{array}{l}\text { Factores de riesgo en el desarrollo de } \\
\text { úlceras de presión y sus implicaciones } \\
\text { en la calidad de vida }\end{array}$ & Lobo $^{29}$ & $\begin{array}{c}2008 \\
\text { Portugal }\end{array}$ & $\begin{array}{l}\text { Estudo } \\
\text { exploratótio } \\
\text { descritivo }\end{array}$ & $\begin{array}{l}\text { Rev Bras } \\
\text { Geriatr } \\
\text { Gerontol }\end{array}$ \\
\hline $\begin{array}{l}\text { Fatores predisponentes para o } \\
\text { desenvolvimento de úlceras por pressão } \\
\text { em idosos institucionalizados }\end{array}$ & $\begin{array}{l}\text { Souza e } \\
\text { Santos }\end{array}$ & $\begin{array}{l}2007 \\
\text { Brasil }\end{array}$ & $\begin{array}{l}\text { Estudo } \\
\text { de coorte } \\
\text { prospectivo }\end{array}$ & $\begin{array}{l}\text { Rev Latino- } \\
\text { Am Enferm }\end{array}$ \\
\hline
\end{tabular}

Quadro 2. Síntese dos principais resultados dos artigos da presente revisão integrativa. Recife, Pernambuco, Brasil, 2017.

\begin{tabular}{|c|c|c|}
\hline Referências & Locais & Principais variáveis relacionadas aos fatores predisponentes \\
\hline 12 & Hospital & DCNTs, IMC abaixo da média, uso de cateter urinário e anemia \\
\hline 20 & Hospital & $\begin{array}{l}\text { IMC acima da média, IMC abaixo da média, alterações } \\
\text { na pele, presença de edema, déficit respiratório, circulatório, } \\
\text { sensorial, nutricional, incontinência urinária, redução } \\
\text { da mobilidade, DCNTs, anemia e uso de medicamentos }\end{array}$ \\
\hline 16 & Hospital & $\begin{array}{l}\text { Baixa pressão sistólica, longo tempo de } \\
\text { permanência no hospital e uso de medicação }\end{array}$ \\
\hline 22 & Hospital & Déficit nutricional e mental \\
\hline 10 & Hospital & $\begin{array}{c}\text { DCNTs, uso de medicação, turgor e elasticidade diminuídos, } \\
\text { redução da mobilidade, confusão mental, depressão, } \\
\text { infecção hospitalar e desnutrição }\end{array}$ \\
\hline 23 & Hospital & Idade avançada, tempo de permanência no hospital \\
\hline 25 & Hospital & $\begin{array}{l}\text { Idade avançada, uso de medicação, tempo de permanência } \\
\text { hospitalar, imobilidade, lesão da medula espinhal, pacientes } \\
\text { cirúrgicos, muitos procedimentos diagnósticos e terapêuticos }\end{array}$ \\
\hline
\end{tabular}


Quadro 2. Continuação.

\begin{tabular}{|c|c|c|}
\hline Referências & Locais & Principais variáveis relacionadas aos fatores predisponentes \\
\hline 29 & Hospital & $\begin{array}{l}\text { Incapacidade de movimentação, déficit neurológico, perda de } \\
\text { sensibilidade, idade avançada e nutrição desequilibrada }\end{array}$ \\
\hline 24 & $\begin{array}{c}\text { Hospital e } \\
\text { instituição } \\
\text { de longa } \\
\text { permanência } \\
\text { para idosos }\end{array}$ & $\begin{array}{l}\text { DCNTs, anemia, uso de cateter urinário, baixo IMC e albumina, disfunção } \\
\text { cognitiva e da consciência; uso de medicamentos e idade avançada }\end{array}$ \\
\hline 27 & $\begin{array}{l}\text { Instituição } \\
\text { de longa } \\
\text { permanência } \\
\text { para idosos }\end{array}$ & $\begin{array}{l}\text { Idade avançada, DCNTs, alteração da pele, imobilidade associada à } \\
\text { instabilidade motora, adelgaçamento da pele e uso de medicamentos }\end{array}$ \\
\hline 03 & $\begin{array}{l}\text { Instituição } \\
\text { de longa } \\
\text { permanência } \\
\text { para idosos }\end{array}$ & $\begin{array}{l}\text { Uso de medicação, pouca habilidade de atividades } \\
\text { da vida diária, função cognitiva, DCNTs, défícit de recursos } \\
\text { humanos, déficit de nutrição, baixo nível de albumina }\end{array}$ \\
\hline 15 & $\begin{array}{l}\text { Instituição } \\
\text { de longa } \\
\text { permanência } \\
\text { para idosos }\end{array}$ & Obesos \\
\hline 30 & $\begin{array}{l}\text { Instituição } \\
\text { de longa } \\
\text { permanência } \\
\text { para idosos }\end{array}$ & $\begin{array}{l}\text { Idade avançada, distúrbio urinário, cardiovascular, respiratório, } \\
\text { neurológico e dermatológico, umidade, nutrição, fricção e cisalhamento }\end{array}$ \\
\hline 26 & $\begin{array}{l}\text { Instituição } \\
\text { de longa } \\
\text { permanência } \\
\text { para idosos }\end{array}$ & Nutrição prejudicada, atividade reduzida, fricção e cisalhamento \\
\hline 17 & $\begin{array}{l}\text { Serviço } \\
\text { domiciliar }\end{array}$ & $\begin{array}{l}\text { Déficit cognitivo, comprometimento de atividades da vida diária, } \\
\text { DCNTs, idade avançada e uso de medicação }\end{array}$ \\
\hline 18 & $\begin{array}{l}\text { Serviço } \\
\text { domiciliar }\end{array}$ & $\begin{array}{l}\text { DCNTS, audição ou visão prejudicada, incontinência } \\
\text { urinária ou fecal, declínio da capacidade funcional, } \\
\text { uso de medicação, limitação física e déficit cognitivo }\end{array}$ \\
\hline 19 & $\begin{array}{l}\text { Serviço } \\
\text { domiciliar }\end{array}$ & $\begin{array}{l}\text { Idade avançada, percepção sensorial diminuída, } \\
\text { umidade, fricção e cisalhamento da pele, atividade } \\
\text { e mobilidade diminuídas e nutrição deficiente }\end{array}$ \\
\hline 02 & $\begin{array}{l}\text { Serviço } \\
\text { domiciliar }\end{array}$ & Mobilidade diminuída, déficit nutricional e incontinência urinária \\
\hline 21 & $\begin{array}{l}\text { Serviço } \\
\text { domiciliar }\end{array}$ & Déficit nutricional, comprometimento cognitivo e dependência física \\
\hline 14 & $\begin{array}{l}\text { Serviço } \\
\text { domiciliar e } \\
\text { hospital }\end{array}$ & $\begin{array}{l}\text { Idade avançada, dependência para as atividades básicas de vida } \\
\text { diárias, redução da mobilidade e comprometimento cognitivo }\end{array}$ \\
\hline 13 & $\begin{array}{l}\text { Centro de } \\
\text { reabilitação }\end{array}$ & Baixo IMC, nutrição, redução da movimentação e alterações na a pele \\
\hline
\end{tabular}


divergência, alguns com predominância de risco no sexo feminino $^{2,13,10,17-21,24,30}$ e outros no masculino ${ }^{3,25}$.

Em relação ao avanço na idade, observou-se que nove artigos trouxeram essa variável entre as relacionadas aos fatores predisponentes para desenvolvimento das LP. Estudo mostra que o paciente com idade adiantada é fator predisponente para o desenvolvimento de LP decorrente das modificações surgidas na pele e nos tecidos subcutâneos provenientes do próprio envelhecimento, evidenciadas por alterações cardiocirculatórias ocasionadas pelas doenças crônico-degenerativas ${ }^{25}$.

Um estudo que avaliou a idade dos pacientes em relação a escores da Escala de Braden e ao aparecimento de LP revelou que a partir dos 70 anos ocorrem alterações, pois a partir dessa idade a pontuação da escala é reduzida devido à relação do aparecimento do agravo ${ }^{28}$.

Para que ocorra um envelhecimento ativo é necessária uma combinação de fatores como idade, sexo, educação, sustentação da habilidade funcional, ou seja, o ser humano precisa ser inserido em um ambiente que preserve as suas condições de saúde, mesmo quando são comprometidos por doenças crônicas não transmissíveis (DCNT). A Organização das Nações Unidas (ONU), em suas políticas públicas para o envelhecimento, preconiza que o envelhecer é um processo ativo, sendo prioritário dar ênfase à qualidade de vida das pessoas com o intuito de ofertar um acesso à saúde digno e igualitário ${ }^{25}$.

Observa-se que os diagnósticos mais citados em relação ao desenvolvimento das LP foram: acidentes vasculares cerebrais, hipertensão arterial sistêmica, diabetes mellitus, cardiopatias, neuropatias e pneumopatias, todos agrupados na categoria das DCNT, para assim ter um melhor entendimento. Essa variável foi encontrada em oito artigos $3,10,12,17,18,20,24,27$.

O paciente idoso acometido de doença crônica pode precipitar mudanças na circulação sanguínea, que levam à diminuição do nível de oxigenação, fator essencial para o processo de cicatrização. Desse modo, esses pacientes podem apresentar retardos na cicatrização da pele, além da redução da sua resistência às lesões pela fragilidade advinda das referidas alterações ${ }^{15}$.

Foi relatado em um estudo, que avaliou a incidência de comorbidades, que pacientes com doença de Alzheimer, acidente vascular cerebral e Parkinson têm uma maior predisposição para o surgimento de LP. Destaca-se a prevalência da hipertensão arterial com 67,8\%, seguida de cardiopatia (30,1\%), diabetes mellitus (27,3\%), Parkinson (20,2\%), Alzheimer (17\%) e acidente vascular cerebral $(14,2 \%)^{18}$.

$\mathrm{O}$ paciente que permanece por longo tempo no leito, por causa de alguma doença que tenha causado um déficit cognitivo moderado ou grave, tem uma maior predisposição ao desenvolvimento de LP, uma vez que o grau de acometimento da cognição interfere na capacidade de movimentação ${ }^{18}$. O déficit cognitivo e o comprometimento neurológico foram vistos em oito artigos desta revisão 3,14,17,18,21,25,29,30. Estudos mostram que o comprometimento de atividades da vida diária é significativo para o surgimento de LP, já que a redução da capacidade funcional está associada à diminuição da mobilidade, variável vista em nove artigos ${ }^{2,10,13,14,19,20,25,27,29}$.

Não obstante, o progresso da demência é um fato natural que inclui mobilidade prejudicada, podendo ser acompanhada de infecções, febres, pneumonia aspirativa e alterações alimentares que propiciam o aparecimento de $\mathrm{LP}^{12}$.

No que se refere à variável alteração da pele, sete artigos $^{13,20,19,20,27,29,30}$ trazem a relação com o desenvolvimento de LP. Um estudo ${ }^{21}$ identificou que a maioria (60\%) dos idosos com pele saudável não apresentava risco para desenvolver LP. Os idosos com a pele muito fina e pele seca, 26 (28,3\%) e $9(34,8 \%)$, respectivamente, tinham alto risco. Quando foram analisados em relação ao edema, 16 (17,3\%) o apresentavam, 4 (25\%) um com altíssimo risco e 5 (31,3\%) com um alto risco. Quanto à pele úmida e pegajosa, totalizava um (1,1\%) artigo, bem como os de pele quebradiça/marcada, um (1,1\%), porém, todos expressavam alto risco. Por fim, entre os que tinham pele descorada, $10(10,9 \%)$ e 4 (40\%) apresentavam alto risco, e 2 (20\%), altíssimo risco para LP. Diante desse cenário, percebe-se que a pele com alteração, ou seja, não saudável, é um fator predisponente importante para LP. Dessa forma, ressalta-se a importância do cuidado dos profissionais de saúde a esses pacientes em relação à prevenção das LP.

Outro ponto importante foi observado em nove artigos $^{3,10,16-18,20,24,25,27}$ que associam o uso de medicamentos e a ocorrência de LP nos idosos. Observa-se que aqueles com mobilidade física prejudicada pela condição de adoecimento e que fazem uso de medicação contínua têm predisposição para o desenvolvimento de $\mathrm{LP}^{25}$.

Vale salientar que algumas medicações podem influenciar na cicatrização das lesões. O resultado do estudo demonstra que os efeitos de alguns medicamentos podem atrapalhar o processo de cicatrização das feridas e trazer consequências negativas para a pele, como o aparecimento de lesões e outras doenças cutâneas ${ }^{26}$.

Outro estudo demonstrou que o uso contínuo de medicações pode ocasionar o surgimento de LP em idosos institucionalizados que apresentam mobilidade física lesada devido a sequelas de doenças ${ }^{25}$. 
Muitos pacientes idosos que fazem o uso de medicação contínua apresentam alterações significantes em respostas aos fármacos. A posologia da medicação também pode sofrer alterações em consequência da incidência crescente de doença com o envelhecimento e da tendência à polifarmácia para os idosos em LP. Alguns medicamentos como anti-inflamatórios alteram a resposta inflamatória do processo de cicatrização, enquanto imunossupressores, quimioterápicos e radioterapia modificam a imunidade do organismo, afetando e reduzindo a cicatrização ${ }^{25}$.

Além desses medicamentos, existem os psicotrópicos, que podem desencadear diversas alterações na pele, consideradas fatores predisponentes à manifestação das LP. Sabe-se que a insulina e os hipoglicemiantes orais também podem estimular o aparecimento de alterações na pele ${ }^{26}$.

Dessa forma, o uso contínuo de medicamentos pode contribuir para o desenvolvimento das LP. As medicações que causam sedação interferem na mobilização do idoso, e os hipotensores diminuem o fluxo sanguíneo e a perfusão tecidual, aumentando a suscetibilidade do individuo às $\mathrm{LP}^{25}$.

Em 11 artigos $2,3,10,13,19-21,24,26,29,30$ foi observado o aspecto nutricional relacionado à imobilidade do paciente, visto que a nutrição insuficiente pode influenciar na sua capacidade funcional, havendo interação entre estar acometido por doença e estar desnutrido. Tanto a doença pode causar a desnutrição secundária, como esta pode influenciar adversamente a doença subjacente ${ }^{26}$.

Nesta perspectiva, entre os artigos analisados, três trazem o índice de massa corporal (IMC) abaixo da média ${ }^{12-24}$, um, o IMC acima da média ${ }^{20}$, dois, a diminuição da albumina ${ }^{3,24}$, dois, a anemia ${ }^{12,24}$, e um, a obesidade ${ }^{15}$; e essas foram apontadas como variáveis associadas ao desenvolvimento da LP.

A deficiência de nutrição nos idosos é um fenômeno de doença complexa, podendo ocasionar uma oxigenação de tecido baixa e, consequentemente, uma redução da quantidade de nutrientes, antibióticos e proteínas do tecido, além de reduzir o resultado da imunização do organismo. O processo de envelhecimento e fatores externos ocasionam mudanças na fisiologia humana, tais como prostração prolongada $\mathrm{e}$ incontinência, que acarreta umidade. Essas condições elevam a vulnerabilidade dos idosos a sofrerem diversos tipos de lesões, incluindo as $\mathrm{LP}^{30}$.

Assim sendo, todos os doentes com predisposição a desenvolver LP necessitam realizar uma triagem em relação a sua situação nutricional. Caso comprove que há presença de algum risco de desnutrição, faz-se necessário realizar uma avaliação mais complexa por um nutricionista, com intuito de oferecer apoio nutricional de acordo com a deficiência de cada indivíduo ${ }^{26}$.

Sendo assim, o uso de protocolos de identificação dos fatores de risco, prevenção e tratamento de LP é uma ferramenta da sistematização da assistência de enfermagem, na medida em que qualifica o cuidado prestado, repercutindo no indicador de melhor qualidade assistencial do trabalho da enfermagem, por meio da redução da incidência de LP, visto que a ocorrência é um indicador do cuidado prestado ${ }^{31}$.

Os cuidados com a integridade da pele e tecidos são elementos da prática do enfermeiro, o que inclui a prevenção das LP. Isso requer uma avaliação dos pacientes com identificação de riscos para esse tipo de lesão e, consequentemente, implementação de intervenções de enfermagem ${ }^{32,33}$ para prevenir complicações que podem comprometer o estado de saúde e a qualidade de vida dos mesmos. A identificação dos pacientes em risco para LP é essencial à implantação de ações preventivas, desde o momento da internação até a sua alta ${ }^{33}$. Um dos instrumentos de predição de risco para LP mais amplamente utilizado por enfermeiros é a Escala de Braden ${ }^{34}$.

Quanto ao cenário das investigações, nove foram realizadas em hospitais, seis em instituições de longa permanência, seis em serviços domiciliares e uma em centro de reabilitação.

\section{CONCLUSÃO}

Neste estudo foi possível observar que houve predominância de risco no sexo feminino, e que em nove artigos a variável avanço da idade estava relacionada aos fatores predisponentes para desenvolvimento das LP.

Os diagnósticos clínicos mais citados em relação ao desenvolvimento das LP foram: acidentes vasculares cerebrais, hipertensão arterial sistêmica, diabetes mellitus, cardiopatias, neuropatias e pneumopatias. O déficit cognitivo e o comprometimento neurológico foram vistos em oito artigos. Quanto à variável alteração da pele, em sete artigos ela está relacionada ao desenvolvimento de LP e nove artigos associam o uso de medicamentos com a ocorrência de LP nos idosos.

Sobre o aspecto nutricional, 11 artigos avaliaram a relação com a imobilidade do paciente, pois esse parâmetro de nutrição insuficiente pode influenciar sua capacidade funcional. Em relação ao cenário das investigações, nove foram realizadas em hospitais, seis em instituições de 
longa permanência, seis em serviços domiciliares e uma em centro de reabilitação.

Pode-se concluir que as LP constituem um sério problema comumente identificável em idosos no âmbito hospitalar, em cuidado domiciliar e os que permanecem em instituições por muito tempo, portanto, deve ser tema de relevância para a prática clínica e para o cuidado de enfermagem. Sua incidência, além de relacionar-se com a condição clínica do idoso, também reflete a qualidade da assistência prestada por parte dos profissionais de saúde, uma vez que sua prevenção é de fácil execução e baixo custo.
Espera-se que este estudo possa contribuir para os profissionais e para outras pesquisas, uma vez que proporciona o conhecimento de algumas variáveis de risco para LP em idosos e identifica um diagnóstico situacional da temática. Este trabalho, atrelado a um planejamento de ações preventivas, contribui como ferramenta para a construção de um plano de assistência a ser implementado desde a admissão do idoso no hospital até a preparação para a alta, incluindo orientações para o cuidado domiciliar, uma vez que por esse meio é promovido o cuidado do idoso em seu próprio ambiente e não o expõe aos riscos presentes nos hospitais.

\section{REFERÊNCIAS}

1. Cox J. Predictors of pressure ulcers in adult critical care patients. Am J Crit Care [Internet]. 2011 [citado em 04 jan. 2017];20(5):364-75. Disponível em: https://www.ncbi.nlm. nih.gov/pubmed/21885457

2. Lima ACB, Guerra DM. Avaliação do custo do tratamento de úlceras por pressão em pessoas hospitalizados usando curativos industrializados. Ciên Saúde Colet [Internet]. 2011 [citado em 04 jan. 2017];16(1):267-77. Disponível em: http://www.scielo.br/scielo.php?script=sci_arttext\&pid $=$ S1413-81232011000100029

3. Freitas MC, Medeiros ABF, Guedes MVC, Almeida PC, Galiza FT, Nogueira JM. Úlcera por pressão em idosos institucionalizados: análise da prevalência e fatores predisponentes. Rev Gaúcha Enferm [Internet]. 2011 Mar [citado em 04 jan. 2017];32(1):143-50. Disponível em: http://www.scielo.br/scielo.php?script=sci_arttext\&pid =S1983-14472011000100019

4. Makai P, Koopmanschap M, Bal R, Nieboer A. Cost effectiveness of a pressure ulcer quality collaborative. Cost Eff Resour Alloc [Internet]. 2010 [citado em 07 jan. 2017];8:11. Disponível em: https://www.ncbi.nlm.nih.gov/ pubmed/20515473

5. Instituto Brasileiro de Geografia e Estatística. Síntese de indicadores sociais: uma análise das condições de vida da população brasileira. Rio de Janeiro: IBGE; 2010.

6. Ortolan MCAB, Biondo-Simões MLP, Baroni ERV, Auersvald A, Auersvald LA, Montemor Netto MR, et al. Influence of aging on the skin quality of white-skinned women: the role of collagen, elastic material density, and vascularization. Rev Bras Cir Plást [Internet]. 2013 [citado em 08 jan. 2017];28(1):41-8. Disponível em: http://www.scielo.br/pdf/ rbcp/v28n1/08.pdf

7. Quirino DES, Faustino AM, Freitas RO, Oliveira AB, Medved IV. Fatores de Risco para o Desenvolvimento de Úlcera por Pressão em Unidade de Internação Clínica. Revista Estima [Internet]. 2014 [citado em 08 jan. 2017];12(4). Disponível em: https://www. revistaestima.com.br/index.php/estima/article/view/99

8. Oliveira SKP, Queiroz APO, Matos DPM, Moura AF, Lima FET. Temas abordados na consulta de enfermagem: revisão integrativa da literatura. Rev Bras Enferm [Internet]. 2012 [citado em 10 jan. 2017];65(1):155-61. Disponível em: http://www.scielo.br/scielo.php?script=sci_arttext\&pid =S0034-71672012000100023

9. Alves AGP, BorgesJWP, Brito MA. Avaliação do risco paraúlcera por pressão em unidades de terapia intensiva: uma revisão integrativa. Rev Pesq Cuidado Fundam [Internet]. 2014 Abr./ Jun. [citado em 10 jan. 2017];6(2):793-804. Disponível em: http://www.redalyc.org/pdf/5057/505750622036_2.pdf

10. Vieira CPB, Sá MS, Madeira MZA, Luz MHBA. Caracterização e fatores predisponentes para úlceras por pressão na pessoa idosa hospitalizada. Rev Rene [Internet]. 2014 [citado em 10 jan. 2017];15(4):650-8. Disponível em: http://www.revistarene. ufc.br/revista/index.php/revista/article/view/1777/pdf

11. Soares CB, Hoga LAK, Peduzzi M, Sangaleti C, Yonekura T, Silva DRAD. Integrative review: concepts and methods used in nursing. Rev Esc Enferm USP [Internet]. 2014 [citado em 10 jan. 2017];48(2):329-39. Disponível em: http://www.scielo. br/pdf/reeusp/v48n2/0080-6234-reeusp-48-02-335.pdf

12. Jaul $E$, Calderon-Margalit R. Systemic factors and mortality in elderly patients with pressure ulcers. Int Wound J [Internet]. 2015 [citado em 13 jan. 2017];12(3):254-59. Disponível em: http://onlinelibrary.wiley.com/doi/10.1111/iwj.12086/full

13. Strazzieri-Pulido KC, Peres GRP, Campanili TCGF, Santos VLCG. Prevalência de lesão por fricção e fatores associados: revisão sistemática. Rev Esc Enferm USP [Internet]. 2015 [citado em 13 jan. 2017];49(4):674-80. Disponível em: http://www.scielo.br/pdf/reeusp/v49n4/pt_0080-6234reeusp-49-04-0674.pdf

14. Akca NK, Aydin G, Gümüs K. Pressure Ulcers and Their Associated Factors in Nursing Home Inmates. I Coll Physicians Surg Pak [Internet]. 2015 [citado em 14 jan. 2017];25(1):27-30. Disponível em: https://www.jcpsp.pk/ archive/2015/Jan2015/08.pdf

15. Cai $S$, Rahman $M$, Intrator O. Obesity and pressure ulcers among nursing home residents. Med Care [Internet]. 2013 [citado em 14 jan. 2017];51(6):478-86. Disponível em: https://www.ncbi.nlm.nih.gov/pmc/articles/PMC3654390/

16. Man SP, Au-Yeung TW. Hypotension is a risk factor for new pressure ulcer occurrence in older patients after admission to an acute hospital. J Am Med Dir Assoc [Internet]. 2013 [citado em 14 jan. 2017];14(8):627.e1-e5. Disponível em: http://www. jamda.com/article/S1525-8610(13)00256-9/abstract?cc=y 
17. Freitas JDPC, Alberti LR. Aplicação da Escala de Braden em domicílio: incidência e fatores associados a úlcera por pressão. Acta Paul Enferm [Internet]. 2013 [citado em 14 jan. 2017];26(6):515-21. Disponívelem:http://www.scielo.br/scielo. php?script=sci_arttext\&pid=S0103-21002013000600002

18. Aguiar ESS, Soares MJGO, Caliri MHL, Costa MML, Oliveira SHS. Avaliação da capacidade funcional de idosos associada ao risco de úlcera por pressão. Acta Paul Enferm [Internet]. 2012 [citado em 16 jan. 2017];25(1):94-100. Disponível em: http://www.scielo.br/scielo.php?pid=\$010321002012000800015\&script=sci_arttext\&tlng=pt

19. Moraes GLA, Araújo TM, Caetano JA, Lopes MVO, Silva MJ. Avaliação de risco para úlcera por pressão em idosos acamados no domicílio. Acta Paul Enferm [Internet]. 2012 [citado em 16 jan. 2017];25(1):7-12. Disponível em: http:// www.scielo.br/pdf/ape/v25nspe1/pt_02

20. Fernandes MDGM, Costa KNDFM, Santos SRD, Pereira MA, Oliveira DST, Brito SDS. Risco para úlcera por pressão em idosos hospitalizados: aplicação da Escala de Waterlow. Rev Enferm UERJ [Internet]. 2012 [citado em 16 jan. 2017];20(1):56-60. Disponível em: http://www.e-publicacoes. uerj.br/index.php/enfermagemuerj/article/view/3977

21. Sancho À, Albiol R, Mach N. Relación entre el estado nutricional y el riesgo de presentar úlceras por presión en pacientes incluidos en el programa de atención domiciliaria. Atención Primaria [Internet]. 2012 [citado em 17 jan. 2017];44(10):586-94. Disponível em: http://www. sciencedirect.com/science/article/pii/S0212656711004173

22. Baumgarten M, Rich SE, Shardell MD, Hawkes WG, Margolis DJ, Langenberg P, et al. Care-Related Risk Factors for Hospital-Acquired Pressure Ulcers in Elderly Adults with Hip Fracture. J Am Geriatr Soc [Internet]. 2012 [citado em 17 jan. 2017];60(2):277-83. Disponível em: https://www.ncbi.nlm. nih.gov/pubmed/22332674

23. Creutzberg M, Aguilera NCF, Cardoso PC, Barbosa TL, Ceolin LD, Stein K, et al. Fatores de risco para úlceras de pressão em idosos de Unidade de Terapia Intensiva. Enferm Foco [Internet]. 2011 [citado em 19 jan. 2017];2(2). Disponível em: http://revista.portalcofen.gov.br/index.php/enfermagem/ article/view/112

24. Shahin ES, Meijers JMM, Schols JMGA, Tannen A, Halfens RJG, Dassen T. The relationship between malnutrition parameters and pressure ulcers in hospitals and nursing homes. Nutrition [Internet]. 2010 [citado em 20 jan. 2017];26(9):886-9. Disponível em: http://www.sciencedirect. com/science/article/pii/S0899900710000547

25. Baumgarten M, Margolis DJ, Localio AR, Kagan SH, Lowe RA, Kinosian $\mathrm{B}$, et al. Extrinsic risk factors for pressure ulcers early in the hospital stay: a nested case - control study. J Gerontol A Biol Sci Med Sci [Internet]. 2008 [citado em 20 jan. 2017];63(4):408-13. Disponível em: http://biomedgerontology. oxfordjournals.org/content/63/4/408.short
26. Lahmann NA, Tannen A, Dassen T, Kottner J. Friction and shear highly associated with pressure ulcers of residents in long-term care - Classification Tree Analysis (CHAID) of Braden items. J Eval Clin Pract [Internet]. 2011 [citado em 22 jan. 2017];17(1):168-73. Disponível em: https://www.ncbi. nlm.nih.gov/pubmed/20831665

27. Kwong EWY, Pang SMC, Aboo GH, Law SSM. Pressure ulcer development in older residents in nursing homes: influencing factors. J Adv Nurs [Internet]. 2009 [citado em 22 jan. 2017];65(12):2608-20. Disponível em: https://www.ncbi. nlm.nih.gov/pubmed/19824910

28. Eleonora GWK, Gissela PFC. Riesgos a úlceras por presión según Escala Norton en una población de ancianos de un servicio domiciliario de Lima. Rev Enferm Herediana [Internet]. 2008 [citado em 24 jan. 2017];1(1):26-32. Disponível em: http:// http://faenf.cayetano.edu.pe/images/ pdf/Revistas/2008/enero/v1n1ao4.pdf

29. Lobo A. Factores de riesgo en el desarrollo de úlceras de presión y sus implicaciones en la calidad de vida. Rev Bras Geriatr Gerontol [Internet]. 2008 [citado em 24 jan. 2017];11(3):405-18. Disponível em: http://www.scielo.br/ pdf/rbgg/v11n3/1981-2256-rbgg-11-03-00405.pdf

30. Souza DMST, Santos VLCG. Fatores predisponentes para o desenvolvimento de úlceras por pressão em idosos institucionalizados. Rev Latino-Am Enferm [Internet]. 2007 [citado em 24 jan. 2017];15(5):958-64. Disponível em: http:// www.scielo.br/pdf/rlae/v15n5/pt_v15n5a11

31. Menegon DB, Bercini RR, Brambila MI, Scola ML, Jansen MM, Tanaka RY. Implantação do protocolo assistencial de prevenção e tratamento de úlcera de pressão no Hospital de Clínicas de Porto Alegre. Rev HCPA [Internet]. 2007 [citado em 24 jan. 2017];27(2):61-4. Disponível em: http:// www.seer.ufrgs.br/\%EE\%80\%80hcpa\%EE\%80\%81/article/ viewFile/2031/1174

32. Schindler CA, Mikhailov TA, Kuhn EM, Christopher J, Conway $P$, Ridling $D$, et al. Protecting fragile skin: nursing interventions to decrease development of pressure ulcers in pediatric intensive care. Am J Crit Care [Internet]. 2011 [citado em 24 jan. 2017];20(1):26-34. Disponível em: https://www.ncbi.nIm. nih.gov/pubmed/21196569

33. Bavaresco $T$, Lucena AF. Nursing intervention classifications (NIC) validated for patients at risk of pressure ulcers. Rev Latino Am Enferm [Internet]. 2012 [citado em 24 jan. 2017];20(6):1109-16. Disponível em: http://www.scielo.br/scielo.php?script=sci_arttext\&pid =S0104-11692012000600013

34. Menegon DB, Bercini RR, Santos CT, Lucena AF, Pereira AGS, Scain SF. Análise das subescalas de Braden como indicativos de risco para úlcera por pressão. Texto Contexto Enferm [Internet]. 2012 [citado em 24 jan. 2017];21(4):854-61. Disponível em: http://www.scielo.br/scielo.php?script=sci_ar ttext\&pid=S0104-07072012000400016 